\title{
Stable Adaptive Control of Manipulators with Improved Transients via Supervision of the Free-Design Parameters and Sampling Period
}

\author{
M. De La Sen, A. Almansa, J. C. Soto and A. J. Garrido \\ Instituto de Investigación y Desarrollo de Procesos \\ Leioa (Bizkaia). Aptdo. 644 de Bilbao \\ SPAIN
}

\begin{abstract}
An adaptive control scheme for mechanical manipulators is proposed. The control loop consists of a network for learning the robot's inverse dynamics and on-line generating the control signal. Some simulation results are provided to evaluate the design. A supervisor is used to improve the performances of the system during the adaptation transients. The supervisor exerts two supervisory actions. The first one consists of updating the free-design adaptive controller parameters so that the value of a quadratic loss function is maintained sufficiently small. The second supervisory action consists of an on-line adjustment of the sampling period within an interval centered at its nominal value.
\end{abstract}

Keywords:- Adaptive control, adaptation transients, supervised adaptive control

\section{Introduction}

The problem of designing adaptive control laws for rigid robot manipulators has interested researchers for many years. The development of effective adaptive controllers represents an important step towards highprecision robotic applications. In recent years, adaptive control results for robotic systems have included rigorous stability analysis (see, for instance, [1-5]). It is important the achievement of good transient performances when synthesizing adaptive control laws. Particular useful tools for that purpose are the on-line updating of the free parameters of the adaptation algorithm and the on-line generation of the sampling period so that the tracking error be improved during the transient. In this paper, an adaptive control scheme for mechanical manipulators is presented that takes advantage of the relationships between adaptive and neural controllers. The synthesized controller involves the use of a supervisor to improve the transient performances since such a strategy was proved to be useful in classical problems of adaptive control to improve the adaptation transients, [6-7]. The proposed supervisory scheme, which is the main contribution of this paper, consists of two major actions, namely: (1) An on-line updating procedure of one of the free-design parameters of the estimation algorithm. An optimization horizon including a set of samples including past measurements and, eventually, tracking error predictions is considered. (2) The sampling period is time-varying and generated from an updating sampling law as dependent of the tracking error rate.

\section{Problem Formulation}

The vector equation of motion of an n-link robot manipulator can be written as

$\tau=\mathrm{M}(\Theta) \ddot{\Theta}+\mathrm{V}(\Theta, \dot{\Theta})+\mathrm{G}(\Theta)+\mathrm{F}(\Theta, \dot{\Theta})$

which is is an $\mathrm{nx} 1$ vector of joint torques; $\Theta, \Theta$ and $\Theta$ are the nx 1 vectors of joint positions, speed and accelerations, respectively; $M(\Theta)$ is the nxn mass matrix of the manipulator; $\mathrm{V}(\Theta, \Theta)$ is an $\mathrm{nx} 1$ vector of centrifugal and Coriolis terms; $G(\Theta)$ is an $n x 1$ vector of gravitational terms and $F(\Theta, \Theta)$ is an $n x 1$ vector of friction terms. The equations of motion (1) form a set of coupled nonlinear ordinary differential equations which are quite complex, even for simple manipulators. One of the most widely used techniques to design a trajectory following control system for such a device is the so-called computed-torque control using feedback linearization. The method consists in a nonlinear model-based feedback to compensate for the nonlinearities present in the robot (see Fig. 1). A nonlinear inner loop decouples and linearizes the robot's dynamics in such a way that a linear outer loop can be used to efficiently control the 
resulting linear system to track a desired trajectory $\Theta_{\mathrm{d}}, \ddot{\Theta}_{\mathrm{d}}, \ddot{\Theta}_{\mathrm{d}}$.

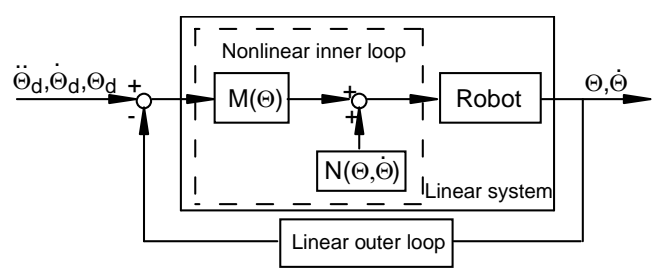

Figure 1. Model-based feedback linearization control scheme

A frequently used computed-torque control scheme is shown in Fig. 1 where $\mathrm{N}(.,$.$) is a nonlinear block$ which includes nonlinear effects on the plant. From the block diagram, the nonlinear, model-based control law is found to be:

$$
\hat{\tau}=\hat{\mathrm{M}}(\ddot{\Theta}) \tau^{\prime}+\hat{\mathrm{V}}(\Theta, \Theta)+\hat{\mathrm{G}}(\Theta)+\hat{\mathrm{F}}(\Theta, \Theta)
$$$$
\text { where } \hat{M}(\Theta), \hat{V}(\Theta, \Theta), \hat{G}(\Theta), \hat{F}(\Theta, \Theta) \text { are }
$$

estimates of $\mathrm{M}(\Theta), \mathrm{V}(\Theta, \Theta), \mathrm{G}(\Theta), \mathrm{F}(\Theta, \Theta)$, respectively, where $\hat{\tau}$ is identical to $\tau$ in (1) with the parameters being replaced by their estimates and $\Theta$ ' has been calculated as:

$$
\tau_{.}^{\prime}=\Theta_{\mathrm{d}}+\mathrm{K}_{\mathrm{V}} \mathrm{E}+\mathrm{K}_{\mathrm{p}} \mathrm{E}
$$

with $K_{V}$ and $K_{p}$ being nxn constant diagonal matrices and the servo error $E$ defined as $E=\Theta_{d}-\Theta$. The error torque becomes:

$$
\begin{aligned}
& \tilde{\tau}_{\mathrm{k}}=\tau_{\mathrm{k}}-\hat{\tau}_{\mathrm{k}} \\
& =\hat{\mathrm{M}}(\Theta) \hat{\mathrm{E}}+\widetilde{\mathrm{M}}(\Theta) \Theta_{\mathrm{d}}+\widetilde{\mathrm{M}}(\Theta) \mathrm{E}-\tilde{\mathrm{M}}(\Theta) \Theta_{\mathrm{d}}(3)
\end{aligned}
$$

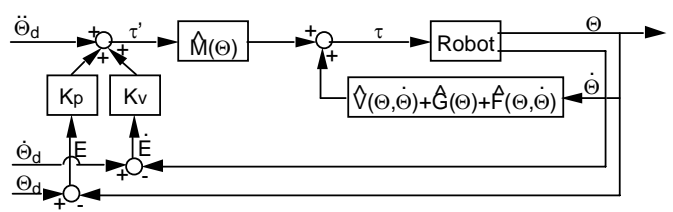

Figure 2.Computed-torque control scheme

(2) using (3) and then substituting in the second-order differential equation obtained from (4) the closedloop dynamics equation is found to be:

$\ddot{\mathrm{E}}+\mathrm{K}_{\mathrm{v}} \dot{\mathrm{E}}+\mathrm{K}_{\mathrm{p}} \mathrm{E}=\hat{\mathrm{M}}^{-1}(\Theta)[\tilde{\mathrm{M}}(\Theta) \ddot{\Theta}+\widetilde{\mathrm{V}}(\Theta, \dot{\Theta})+\widetilde{\mathrm{G}}(\Theta)+\widetilde{\mathrm{F}}(\Theta, \dot{\Theta})]$ where the modeling parametrical errors are ;

$\tilde{\mathrm{M}}(\Theta)=\mathrm{M}(\Theta)-\hat{\mathrm{M}}(\Theta)$

$$
\begin{aligned}
& \tilde{V}(\Theta, \Theta)=V(\Theta, \Theta)-\hat{V}(, \Theta) \\
& \tilde{G}(\Theta)=G(\Theta)-\hat{G}(\Theta) \\
& \tilde{F}(\Theta, \Theta)=F(\Theta, \Theta)-\hat{F}(\Theta, \Theta)
\end{aligned}
$$

If all the robot's parameters are perfectly known, the closed loop equation (5) takes the following linear and decoupled form since the terms in the right-hand side brackets of (5) become zero:

$$
\ddot{E}+K_{v} \dot{E}+K_{p} E=0
$$

so that it becomes clear that a simple suitable selection of $\mathrm{K}_{\mathrm{p}}$ and $\mathrm{K}_{\mathrm{V}}$ can easily regulate the evolution of the servo error. However, although some parameters of a robot are easily measurable, some other effects, such as friction, mass distribution or payload variations cannot, in general, be accurately modeled, and thus the assumption of obtaining negligible modeling errors is quite unrealistic in practice. In these conditions, it looks apparent that some sort of adaptive parameter estimation mechanism should be included in the control loop, so that equation (5) became approximately linear and uncoupled and the servo errors could be asymptotically eliminated.

\section{Adaptive Control Scheme}

The equations of motion (1), although quite complex and nonlinear in general, can be expressed in a linear in the parameters form, since all the potentially unknown parameters (link masses, lengths, friction coefficients, etc.) appear as coefficients of known functions of the generalized coordinates. In an adaptive control system design context, one usually takes the advantage of the above property of linearity in the parameters by rewriting (1) as:

$$
\begin{aligned}
& M(\Theta) \Theta \\
&=
\end{aligned}
$$

where $\mathrm{P}$ is an rxl vector containing the robot's unknown parameters and $\mathrm{W}(\Theta, \Theta, \ddot{\Theta})$ is an nxr matrix of known nonlinear functions, often referred to as regression matrix. In the same way, the $\mathrm{rx} 1$ estimated parameters vector $\hat{\mathrm{P}}$ fulfill

$$
\begin{aligned}
\tilde{M}(\Theta) \ddot{\Theta} & +\tilde{V}(\Theta, \Theta)+\tilde{G}(\Theta)+\widetilde{F}(\Theta, \Theta) \tilde{M}(\Theta) \\
& =W(\Theta, \Theta, \Theta) \tilde{P}
\end{aligned}
$$


where the parameter estimation error $\tilde{\mathrm{P}}$ has been defined as $\tilde{\mathrm{P}}=\mathrm{P}-\hat{\mathrm{P}}$. Figs. 2 and 3 show the adaptive control scheme. The design is a neural extension of the computed-torque control strategy. A two-layered learning network with nxr inputs and $\mathrm{n}$ outputs is used to learn the manipulator's inverse dynamics, so that the control law can be on-line generated. The network's inputs are known nonlinear functions of the system response (more concretely, the elements $\mathrm{w}_{\mathrm{ij}}$ of the regression matrix $\mathrm{W}$ $(\Theta, \Theta, \ddot{\Theta})$ are defined in eqn. (7)), while its outputs are estimates of the input torques:

$$
\hat{\tau}(\mathrm{t})=\hat{\tau}_{\mathrm{k}}=\sum_{\mathrm{i}=1}^{\mathrm{n}} \sum_{\mathrm{j}=1}^{\mathrm{r}} \mathrm{w}_{\mathrm{ij}}(\Theta, \dot{\Theta}, \ddot{\Theta}) \hat{\mathrm{p}}_{\mathrm{j}_{\mathrm{k}}}
$$

for $t \in\left[t_{k}, t_{k+1}\right)$ which is a piecewise constant signal from the zero-order sampling and hold device. Defining the connection weights vector and the estimated torques vector as:

$$
\hat{\mathrm{P}}=\left[\hat{\mathrm{p}}_{1}, \hat{\mathrm{p}}_{2}, \ldots, \hat{\mathrm{p}}_{\mathrm{r}}\right]^{\mathrm{T}} ; \hat{\tau}=\left[\hat{\tau}_{1}, \hat{\tau}_{2}, \ldots, \hat{\tau}_{\mathrm{n}}\right]^{\mathrm{T}}
$$

Eqn. 10 can be expressed in a familiar matrix form:

$$
\hat{\tau}_{k}(t)=\hat{\tau}_{k}=W(\Theta, \Theta, \ddot{\Theta}) \hat{P}_{k}
$$

for $\mathrm{t} \in\left[\mathrm{t}_{\mathrm{k}}, \mathrm{t}_{\mathrm{k}+1}\right)$ where $\hat{\mathrm{P}}_{\mathrm{k}}$ is a parameter vector which is estimated in a discrete-time way, i. e. , it is only updated at sampling instants by the adaptation algorithm. The inverse dynamics is learned as follows:

$\hat{\mathrm{P}}_{\mathrm{k}+1}=\hat{\mathrm{P}}_{\mathrm{k}}+\frac{\mathrm{F}_{\mathrm{k}} \mathrm{W}_{\mathrm{k}}^{\mathrm{T}} \mathrm{E}_{\tau \mathrm{k}}}{\mathrm{c}_{\mathrm{k}}+\mid \mathrm{W}_{\mathrm{k}} \mathrm{F}_{\mathrm{k}} \mathrm{W}_{\mathrm{k}}^{\mathrm{T}} \|}$

$\mathrm{F}_{\mathrm{k}+1}=\frac{1}{\lambda_{\mathrm{k}}}\left(\mathrm{F}_{\mathrm{k}} \frac{\mathrm{F}_{\mathrm{k}} \mathrm{W}_{\mathrm{k}}^{\mathrm{T}} \mathrm{W}_{\mathrm{k}} \mathrm{F}_{\mathrm{k}}}{\mathrm{c}_{\mathrm{k}}+\left\|\mathrm{W}_{\mathrm{k}} \mathrm{F}_{\mathrm{k}} \mathrm{W}_{\mathrm{k}}^{\mathrm{T}}\right\|}\right)$

where $E_{\tau k}$ is the prediction error vector, defined as $\mathrm{E}_{\tau \mathrm{k}}=-\tilde{\tau}_{\mathrm{k}}=\hat{\tau}_{\mathrm{k}}-\tau_{\mathrm{k}}, \mathrm{W}_{\mathrm{k}}($.$) is the$ regression matrix used for updating the parameters, and $\mathrm{F}_{\mathrm{k}}$ is an adaptation gain matrix which satisfies $\mathrm{F}_{0}=\mathrm{F}_{0}^{\mathrm{T}}>0$. The parameter sequence $\lambda_{\mathrm{k}} \in(0,1)$ is the forgetting factor used to update the adaptation gain matrix and $c_{k} \in(0, \infty)$ is a scalar for all $k \geq 0$. Both free parameters of the algorithm have to satisfy the given stability constraints in order to achieve closed-loop stability. The matrix sequence obtained from (12) is positive definite (at the limit it can become semidefinite) and time- decreasing. The norms taken in (12) are the Euclidean norms. The above approach is then used in the simulated example to evaluate the supervision efficiency. If the manipulator's inverse dynamics is correctly learned by the neural network, both nonlinear dynamics cancel each other according to the block diagram shown in Fig. 3. Thus, the closed-loop system turns linear and the closed-loop tracking properties are adjusted with a suitable selection of the proportional and derivative gain matrices $\mathrm{K}_{\mathrm{p}}, \mathrm{K}_{\mathrm{v}}$. This is the same effect obtained using the conventional adaptive control approach described in the previous section.

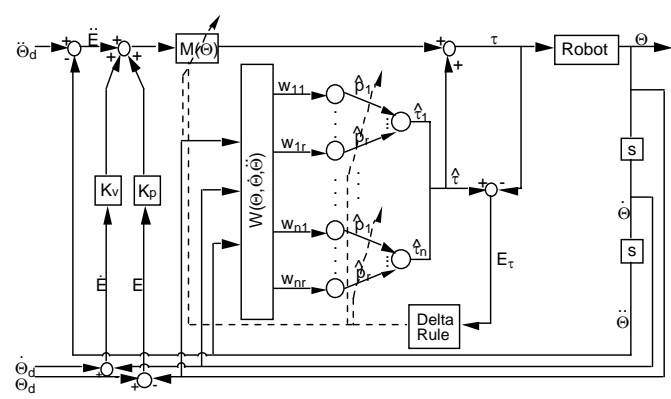

Figure 3. Proposed neural control scheme

\section{Supervisor Design}

\subsection{Heuristic Motivation}

Note, by inspection, that the learning rule associated with the adaptation algorithm (11)-(12) has an adaptation rate highly dependent on the size of the $\mathrm{c}$ $\mathrm{k}$ - updating parameter which is a free- design parameter provided that it is positive and bounded. The adaptation rate is very low when the $\mathrm{c}_{\mathrm{k}}$ sequence takes very large values compared to the square of the regressor norm. A second action of the supervisor is concerned with the on-line choice of the sampling period within an interval centered around a nominal sampling period. The boundary of the variation domain of the sampling period is established according to 'a priori' knowledge about guaranteeing closed- loop stability and a prefixed bandwidth. Other considerations as, for instance, the upper limit of the sampling rate or the achievable performance of the application at hand. The overall supervisor is designed for: (1) An on-line calculation of a free parameter of the adaptation algorithm. (2) A calculation of a time-varying sampling period dependent on the time variation of the tracking error. It is based upon three main rules, namely:

Rule 1: If the tracking error is increasing with respect to preceding samples then decrease 
(increase ) the last value of the sequence of the free - design parameter provided that the previous action at the preceding sample was to increase ( decrease ) the value of such a sequence.

[In other words, if the tracking performance is deteriorating then make an action to correct the supervision philosophy of the last action exerted on the value of the free parameter $f$ the algorithm].

Rule 2 : If the tracking error is decreasing with respect to preceding samples then decrease ( increase ) the last value of the sequence of the free design parameter provided that the previous action was to decrease ( increase) it. [In other words, if the tracking performance is being improved then do not modify the last action exerted on the value of the free parameter ].

$\underline{\text { Rule 3: }}$ Compute a time- varying updated sampling period as being inversely proportional to the absolute value of the tracking error time-derivative within a predefined interval:

$$
\left[\mathrm{T}_{\min }, \mathrm{T}_{\max }\right]=\left[\mathrm{T}_{0}-\Delta \mathrm{T}, \mathrm{T}_{0}+\Delta \mathrm{T}\right]
$$

centred around a prefixed nominal sampling period $\mathrm{T}_{0}$. Thus, the sampling period decreases as the tracking error absolute value grows faster and vice versa,. In Rule $3, \frac{\Delta \mathrm{T}}{\mathrm{T}_{0}}$ has to be small since the discrete parameterization of the plant is time- varying under time- varying sampling. Thus, small variations of the sampling period lead to small deviations of the plant parameterization from time- invariance and the estimation algorithm is still valid in practical situations. This is the philosophy used in this paper to design the admissibility domain of the sampling period. Another useful variation would be, for instance, to use a time- varying whose length decreases asymptotically converging to the nominal sampling period; i.e., $\left[\mathrm{T}_{\text {min }}(\mathrm{k}), \mathrm{T}_{\mathrm{ma}}(\mathrm{k})\right]$ is designed so that $\mathrm{T}_{\text {min }}(\mathrm{k}) \rightarrow \mathrm{T}_{0}$ and $\mathrm{T}_{\max }(\mathrm{k}) \mathbb{T}_{0}$ as $\mathrm{k} \rightarrow \infty$. On the other hand, Rule 3 can also be modified by involving higher- order time - derivatives of the tracking error. The above three actions are completed with the design philosophies:

(1) The sizes of the modifications in the successive values of the $c_{k}$ - sequence of the parameter adaptive algorithm are related to the 'amplitudes' of the improvement or deterioration of the transient performances within the stability constraints for the design parameter $\mathrm{c}_{\mathrm{k}}$ with its values being positive and bounded.
(2) It is better to analyze the transient tracking errors over a finite horizon of preceding samples and, eventually, also on a finite horizon of its future predictions over each current sample in order to include both a correcting and a predictive-correction effects of registered tracking errors to calculate the current value of the sequence of free- design parameters. The use of a unique sample in the supervisory loop would lead, in general, to unsuitable actions when measurement failures arise or when abrupt changes in the control input appear art isolated sampling instants .

\subsection{Supervisory action on a free - design} parameter of the adaptation algorithm

Define the loss function $J_{k}^{\varepsilon}=\sum_{i=k-N_{1}}^{k+N_{2}} \sigma^{|k-i|} \mathbf{E}_{i}^{T} Q_{i} \mathbf{E}_{i}$, for each current $\mathrm{k}$ - th sample as supervisory criterion where $\mathbf{E}()=.\left[\mathrm{E}_{1}(.), \mathrm{E}_{2}(.), \mathrm{E}_{3}(.)\right]^{\mathrm{T}}$ is the tracking error vector, $\mathrm{N}_{1}$ and $\mathrm{N}_{2}$ are, respectively, the sizes of the correction and prediction horizons $[\mathrm{k}$ $-\mathrm{N}_{1}, \mathrm{k}$ ) and [ $\mathrm{k}, \mathrm{k}+\mathrm{N}_{2}$ ] associated with the

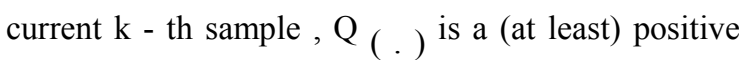
semidefinite weighting matrix and $0<\sigma \leq 1$ is the forgetting factor of the loss function . Note that $\mathbf{E}_{j}$ for $\mathrm{j}>\mathrm{k}$ are predicted tracking errors in the loss function for each $\mathrm{k}$ - th sample. In this paper, the free design parameter in (13) is $\mathrm{c}_{\mathrm{k}}$ which has to belong to an admissibility interval compatible with the stability constraint, i. e. it has to be positive and bounded. The horizon sizes, weighting matrix and forgetting factor of the loss function are chosen by the designer according to the next design criteria : (a) How relatively important each robot articulation is compared to the remaining ones. This idea is relevant top the choice of the $\mathrm{Q}_{(.)^{-}}$matrix. In Fig. 4, the third articulation could be considered more important, if suited, since it has to follow a reference related to the final trajectory for each specific application. If the matrix is chosen as diagonal with positive identical diagonal entries then all the articulations are considered equally relevant and then all the tracking error components are introduced with identical weights in the supervisory loss function.

(b) The relative weight in the loss function given to the more recent measured errors and their next immediate predictions is large compared to the older ones and subsequent future ones, respectively. 
(c) The relative weight in the loss function given to the past tracking errors (correction horizon) compared to the predicted errors (prediction horizon) . The supervisory action over $\mathrm{c}_{\mathrm{k}}$ is described in the following algorithm:

4.3 Supervision of $\mathrm{c}_{\mathrm{k}}$ Step 0 : Define $\left[\mathrm{c}_{\text {min }}, \mathrm{c}_{\text {max }}\right]$ with $\mathrm{c}_{\text {min }}>0$, $\mathrm{c}_{\text {max }}>\mathrm{c}_{\text {min }}>0$ as the admissibility domain for the free parameter $\mathrm{c}_{\mathrm{k}}$ of the adaptation algorithm (13).

Define also the loss function $\mathbf{J}$ according to the above supervisory design criteria (a) to (c).

Initialize $\mathrm{k} \rightarrow 0$.

Step 1 : For each current k-th sampling instant, Make $\bar{c}_{k}=\rho_{k} \| W_{k} F_{k} W_{k}^{\mathrm{T}} \mid+\bar{c}$

$$
\begin{aligned}
& \int \mathrm{c}_{\text {min }} \text { if } \overline{\mathrm{c}}_{\mathrm{k}} \leq \mathrm{c}_{\text {min }} \\
& 1 \\
& \mathrm{c}_{\mathrm{k}}= \begin{cases}\overline{\mathrm{c}}_{\mathrm{k}} & \text { if } \quad \overline{\mathrm{c}}_{\mathrm{k}} \in\left(\mathrm{c}_{\min }, \mathrm{c}_{\text {max }}\right) \\
\mathrm{c}_{\text {max }} & \text { if } \overline{\mathrm{c}}_{\mathrm{k}} \geq \mathrm{c}_{\text {max }}\end{cases} \\
& \rho_{k}=\rho_{k-1}+\operatorname{Min}\left(g_{k}, \operatorname{Int} \operatorname{par}\left[\frac{J_{k}-J_{k-1}}{J_{k-1}}\right]\right) \cdot \operatorname{Sigr}\left[\rho_{k-2}-\rho_{k-1}\right] . \Delta \rho \\
& ; \mathrm{g}_{\mathrm{k}}=\frac{\mathrm{K}-\overline{\mathrm{c}}}{\| \mathrm{W}_{\mathrm{k}} \mathrm{F}_{\mathrm{k}} \mathrm{W}_{\mathrm{k}}^{\mathrm{T}} \mid \Delta \rho} \text { with sign }(0)=0 \text {. }
\end{aligned}
$$

Step 2: Apply the parameter -estimation algorithm of Eqn. (13) and Generate the torque Eqn. ( 2 ). Step 3: $\mathrm{k} \rightarrow \mathrm{k} .1$ and then Go to Step 1. End If the loss function value decreases the supervisory policy has to be kept. The saturation $\mathrm{g}_{\mathrm{k}}$ for the modification of $\Theta_{\mathrm{k}}$ in Step 1 guarantees that $\mathrm{c}_{\mathrm{k}}$ is upper-bounded by a predefined positive design constant $\mathrm{K}$. The small positive constant $\overline{\mathrm{c}}$ is used to avoid division by zero in the parameter estimation (13) when the measurement regressor is zero. The supervisory learning rule also ensures, apart from the above mentioned saturation, that the eventual corrections on the choice of the parameters increase as the efficiency deteriorates.

\subsection{Error Prediction}

The measurements of the loss function in the prediction horizon are calculated by extrapolations of preceding predictions or real measurements by using a Taylor series expansion approximations with finite differences using sampled values according to: $\mathrm{f}_{\mathrm{k}+1} \cong \sum_{\mathrm{i}=0}^{\infty} \frac{\mathrm{T}^{\mathrm{i}} \mathrm{f}_{\mathrm{k}}^{(\mathrm{i})}}{\mathrm{i} !}=\mathrm{f}_{\mathrm{k}}+\left(\mathrm{f}_{\mathrm{k}}-\mathrm{f}_{\mathrm{k}-1}\right)+\frac{1}{2}\left(\mathrm{f}_{\mathrm{k}}-\mathrm{f}_{\mathrm{k}-1}+\mathrm{f}_{\mathrm{k}+2}\right)$ with $T$ being the sampling period for any signal $f(t)$ and the $i$ - th derivative $\mathrm{f}_{\mathrm{k}}^{(\mathrm{i})}$ being defined recursively from $\mathrm{f}_{\mathrm{k}}^{(1)}=\frac{\mathrm{f}_{\mathrm{k}}-\mathrm{f}_{\mathrm{k}-1}}{\mathrm{~T}}$ for $\mathrm{i} \geq 1$. Note that even in the case when the predictions are very rough, this is not very relevant for the supervisory algorithm efficiency because of the saturation effect included in Step 1. In this context, it is suitable to have acceptable predictions of the signs of the next tracking errors for each current sample rather than good estimations of their real values. A simple estimation procedure as the proposed one can be sufficient as shown through simulations in Section 5. 3. It is also important to find an efficient balance between the sizes of the correction and prediction horizons Numerical experimentation involving different sizes of the correction and prediction horizons will help the designer in the choice of their more convenient values.

4.5 Supervisory action on the sampling rate (i. e. On-line updating rule for the time-varying sampling period $T_{k}$ )

The sequence of sampling instants $\left\{\mathrm{t}_{\mathrm{k}}, \mathrm{k} \geq 0\right\}$ is generated as $\mathrm{t}_{\mathrm{k}+1}=\mathrm{t}_{\mathrm{k}}+\mathrm{T}_{\mathrm{k}}$ with $\mathrm{t}_{0}=0$ and $\mathrm{T}_{\mathrm{k}}$ as follows:

$$
\mathrm{T}_{\mathrm{k}}=\left\{\begin{array}{c}
\mathrm{T}_{\text {min }} \text { if } \quad \overline{\mathrm{T}}_{\mathrm{k}} \leq \mathrm{T}_{\text {min }} \\
\overline{\mathrm{T}}_{\mathrm{k}}=\frac{\mathrm{CT}_{\mathrm{k}-1}}{\left|\varepsilon_{\mathrm{k}}-\varepsilon_{\mathrm{k}-1}\right|} \text { if } \mathrm{T} \in\left[\mathrm{T}_{\min }, \mathrm{T}_{\max }\right] \\
\mathrm{T}_{\text {max }} \text { if } \quad \overline{\mathrm{T}}_{\mathrm{k}} \geq \mathrm{T}_{\max }
\end{array}\right.
$$

where a discrete approximation $\overline{\mathrm{T}}_{\mathrm{k}}=\frac{\mathrm{CT}_{\mathrm{k}-1}}{\left|\dot{\varepsilon}\left(\mathrm{t}_{\mathrm{k}}\right)\right|}$ is used by monitoring the tracking error rate and $\mathrm{C}>0$ is an arbitrary real constant. The admissibility interval $\left[\mathrm{T}_{\min }, \mathrm{T}_{\max }\right]$ of the sampling period is selected according to considerations of stability, bandwidth and the requirements on performance of each particular application. The above sampling law is tested in the simulations to evaluate the performance improvement of the sampling rate updating for the transient adaptation. The above sampling law as well as other five updating sampling laws listed below are then comparatively tested in the simulations to evaluate the various improvements caused by the sampling rate adaptation over the basic freeparameter adaptation. The supervisory technique can be also applied to the forgetting factor by making it time-varying with $\lambda_{k} \in(0,1]$ to ensure closed-loop stability of the adaptive scheme. A useful technique is 
to modify the Supervision Algorithm of Section 4.2 to on-line estimate the forgetting factor which has to belong to the admissibility domain $\left[\lambda_{\min }, \lambda_{\max }\right]=[\delta, 1-\delta)$ for some constant $\delta \in(0,1)$ with the change $\overline{\mathrm{c}} \rightarrow \bar{\lambda}>0$ and Step 2 is modified with the replacement $\bar{c}_{\mathrm{k}} \rightarrow \bar{\lambda}_{\mathrm{k}}=\rho_{\mathrm{k}} \lambda_{\mathrm{k}-1}+\bar{\lambda}$ with $\rho_{\mathrm{k}}$ being computed as above and

$$
\lambda_{\mathrm{k}}=\left\{\begin{array}{cc}
\lambda_{\text {min }} & \text { if } \bar{\lambda}_{\mathrm{k}} \leq \lambda_{\text {min }} \\
\bar{\lambda}_{\mathrm{k}} & \text { if } \bar{\lambda}_{\mathrm{k}} \in\left(\lambda_{\min }, \lambda_{\text {max }}\right) \\
\lambda_{\text {max }} & \text { if } \bar{\lambda}_{\mathrm{k}} \geq \lambda_{\text {max }}
\end{array}\right.
$$

Subsequently, the free parameter of the adaptive algorithm $\mathrm{c}_{\mathrm{k}}$ is chosen according to the rule

$$
\mathrm{c}_{\mathrm{k}}=\frac{\lambda_{\mathrm{k}}\left\|\mathrm{W}_{\mathrm{k}} \mathrm{F}_{\mathrm{k}} \mathrm{W}_{\mathrm{k}}^{\mathrm{T}}\right\|}{\mathrm{T}_{\mathrm{r}}\left(1-\lambda_{\mathrm{k}}-\delta\right)}
$$

with $\mathrm{T}_{\mathrm{r}} \geq \operatorname{Trac}\left(\mathrm{F}_{0}\right) \geq \operatorname{Trac}\left(\mathrm{F}_{\mathrm{k}}\right)>0$. Such a rule ensures that the trace of the adaptation matrix remains upperbounded by a prefixed finite bound $\mathrm{T}_{\mathrm{r}}$ for all time in spite of the fact that the adaptation gain matrix is not necessarily time- decreasing.. The closed-loop system is proved to be stable under the above parameter- adaptive law and supervision rules, [7].

\section{Simulation Example}

A planar mechanical manipulator with three revolute joints is considered. We take SI units: $\mathrm{m}_{1}=4.6 ; \mathrm{m}_{2}=2.3 ; \mathrm{m}_{3}=1.0$; $\mathrm{I}_{\mathrm{Zz}}=0.1 ; \quad \mathrm{l}_{1}=\mathrm{l}_{2}=0.5 ; \quad \mathrm{v}_{1}=\mathrm{v}_{2}=\mathrm{v}_{3}=0.5 ; \quad \mathrm{k}_{1}=\mathrm{k}_{2}=\mathrm{k}_{3}=0.5$ where $v_{i}$ and $k_{i}$ are the viscous and Coulomb friction coefficients, respectively. The free-design parameter $\mathrm{c}_{\mathrm{k}}$ and the sampling period are supervised together with values $\Theta_{\mathrm{k}}=1$ in $(13 \mathrm{~b}), \overline{\mathrm{c}}=5, \mathrm{c}_{\mathrm{min}}=5, \mathrm{c}_{\max }=5 \times 10^{7}$ and $\mathrm{c}$ $0=5 \times 10^{6}$ have been chosen in the learning rule (13). In the absence of supervisor, $\mathrm{c}_{\mathrm{k}}=\mathrm{c}_{0}=5 \times 10^{6}$, for all $\mathrm{k} \geq$ 0 . The $\Theta$ weighting matrix of the loss function $\mathrm{J}^{\varepsilon}$ is $Q_{(}$ .) $=\operatorname{Diag}[0.2,0.2,0.2]$ for the samples of the prediction horizon and $\mathrm{Q}_{(\mathrm{f})}=\operatorname{Diag}[0.9,0.9,0.9$ ] for those of the correction horizon; $\sigma=0.5 ; \mathrm{K}=10$ ( Step 2 of the Supervisory Algorithm of Section 4.b) ; $\overline{\mathrm{c}}=5$, $. \rho_{0}=2$ and $\Delta \rho=0.1$. The correction and prediction horizons are chosen with $\mathrm{N}_{1}=5$ and $\mathrm{N}_{2}=2$. The network's connection weights have been initialized as $\hat{\mathrm{p}}_{10}=10, \hat{\mathrm{p}}_{20}=5, \hat{\mathrm{p}}_{30}=1, \hat{\mathrm{p}}_{40}=1$. The values for the proportional and derivative gain matrices $\mathrm{K}_{\mathrm{p}}=\mathrm{K}_{\mathrm{V}}=$ Diag $(100,100,100)$. Acceptable values of the maximum and minimum values of the admissibility interval of the sampling period are until ${ }_{-}^{+} 20 \%$ of its nominal value. The nominal sampling period is $\mathrm{T}_{0}=0.6 \times 10^{-3}$ secs. with variation domain $\left[\mathrm{T}_{\min }, \mathrm{T}_{\max }\right]=[0.5,0.7]$ with $\mathrm{C}=1$. Simulation results about the supervised and unsupervised tracking performance and the control effort are displayed on Figure 4. See [7] for the scheme's design details and exhaustive performed numerical experimentation
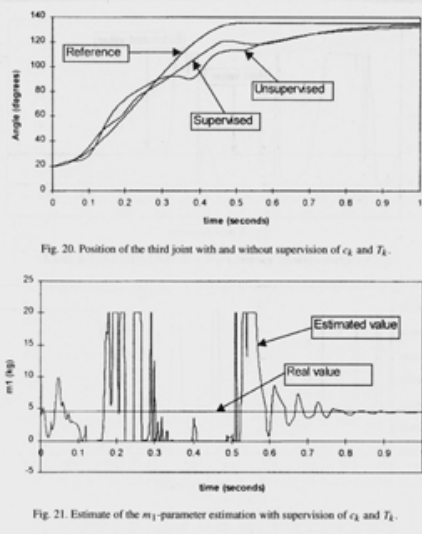

Figure 4. Combined Supervision Performance

\section{ACKNOWLEDGMENT}

The authors are grateful to MCYT and to Basque Government for their partial support of this work through DPI 2006-0714 and S-PEO6UN10-SAIOTEK 2006.

\section{REFERENCES}

[1] Craig, J.J., 1986, Introduction to Robotics, Mechanics and Control, (Reading, Mass.: Addison-Wesley).

[2] Craig, J.J., 1988, Adaptive Control of Mechanical Manipulators, (Reading, Mass.: Addison-Wesley).

[3] Slotine, J.J.E. and LI, W., 1989, "Composite adaptive control of robot manipulators", Automatica, 25, 509-519.

[4] Li, W. and Slotine, J.J.E., 1989, An indirect adaptive controller, Systems and Control Letters, 12, 259-266.

[5] Sadegh, N. and Horowitz, R., 1990, "Stability and robustness analysis of a class of adaptive controllers for robotic manipulators", International Journal of Robotics Research, 9, 74-92.

[6] De la Sen, M., "Adaptive control of time-varying systems with time-varying delays", Dynamics of Continuous, Discrete and Impulsive Systems, Series A: Mathematical Analysis, Vol. 12 (2005), pp. 45- 66, 2005.

[7] De la Sen, M., Miñambres, J.J., Garrido, A.J., Almansa, A. and Soto, J.C. " Basic theoretical results for expert systems. Application to the supervision of adaptation transients in planar robots", Artificial Intelligence, Vol. 152, No. 4 (2004), pp. 173-211, 2004. 